\title{
Segmented waveguides in thin silicon-on-insulator
}

\author{
Michael Hochberg, Tom Baehr-Jones, Chris Walker, and Jeremy Witzens \\ California Institute of Technology, 1200 East California Boulevard, Pasadena, California 91125 \\ Lawrence C. Gunn \\ Luxtera, Inc., 1819 Aston Avenue, Carlsbad, California 92008 \\ Axel Scherer \\ California Institute of Technology, 1200 East California Boulevard, Pasadena, California 91125
}

Received June 4, 2004; revised manuscript received August 30, 2004; accepted October 10, 2004

\begin{abstract}
We have developed new silicon-on-insulator waveguide designs for simultaneously achieving both low-loss optical confinement and electrical contacts, and we present a design methodology based on calculating the Bloch modes of such segmented waveguides. With this formalism, waveguides are designed in a single thin layer of silicon-on-insulator to achieve both optical confinement and minimal insertion loss. Waveguides were also fabricated and tested, and the measured data were found to closely agree with theoretical predictions, demonstrating input insertion loss and propagation loss better than $0.1 \mathrm{~dB}$ and $-16 \mathrm{~dB} / \mathrm{cm}$, respectively. (C) 2005 Optical Society of America
\end{abstract}

OCIS codes: $220.3740,230.7390,130.1750$.

\section{INTRODUCTION}

Recently, low-loss single-mode waveguides in thin siliconon-insulator (SOI) have been demonstrated ${ }^{1}$ with $<-7 \mathrm{~dB} / \mathrm{cm}$ of propagation loss. There are several advantages to working in such a material; in particular, the thin geometry is helpful in obtaining high field concentrations in the waveguide cladding, a useful attribute for the construction of optical chemical sensors or silicon waveguides clad with electro-optic polymers. Typically, the functionalization material in such devices resides in the waveguide cladding, and a large modal overlap with the deposited cladding layer is desirable. One of the outstanding problems of this geometry, however, is the difficulty of establishing an electrical contact with the waveguide without perturbing the optical mode. This is particularly important when a dc or rf electrical field is to be applied directly onto the waveguiding region. Such a field can be used, for instance, to induce modulation through an electro-optic index shift in the cladding. ${ }^{2}$ As the lateral dimensions of such single-mode SOI waveguides are necessarily $\sim 500 \mathrm{~nm}$ for a $120 \mathrm{~nm}$ thin silicon SOI layer to ensure single-mode propagation, it is challenging to generate the field amplitudes desired at the surface of the waveguide without electrically contacting the waveguide. Substantial research has been done previously on segmented waveguides in low-index-contrast, large-mode-volume geometries. $^{3-5}$

The SOI geometry that we use in this study was chosen to have low waveguide loss and relatively large field concentrations outside the core of the waveguide. It consists of $120 \mathrm{~nm}$ of silicon (index 3.5) atop a $1.4 \mu \mathrm{m}$ layer of silicon dioxide (index 1.43), which rests on the silicon handle.
The structures were clad in polymethyl methacrylate ( $n$ =1.43). The optical mode that such a waveguide supports is primarily polarized horizontally and is essentially isolated from the substrate by the oxide layer, although it can be shown that some loss induced by tunneling leakage into the substrate will occur. ${ }^{6}$ The dispersion plot of the fundamental mode and modal concentration are detailed in Fig. 1.

The definition of an electrical contact on such a waveguide is particularly difficult in that the waveguide is both electrically and optically isolated on all sides by silicon dioxide cladding. The introduction of an electrical contact leads to a significant interruption in the waveguide symmetry and therefore a large scattering loss. Here we show a remedy for this problem by constructing segmented waveguides as shown in Fig. 2 .

In this figure the essential features of the segmented waveguide approach are detailed. The electrical contact is defined as a lateral grating or a planar extension that is lithographically defined during the same lithographic step as the waveguide definition etch. The optical properties of this geometry are strongly dependent on the periodicity and duty cycle. (We consider the duty cycle to be the fraction of the period that contains the segment; thus a duty cycle of 0.7 on a period of $1 \mu \mathrm{m}$ indicates segments of silicon that are $0.7 \mu \mathrm{m}$ long.) In theory the lateral silicon strips in Fig. 2 continue forever, but, as we will see, for properly chosen periodicities these can be terminated after a relatively short isolation distance. If a low-loss, propagating optical mode exists for a particular design, it is possible to achieve both the desired low-loss optical guiding and lateral electrical contact to the waveguide. 

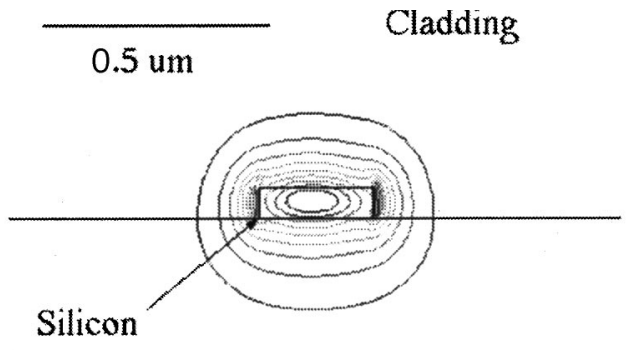

Silicon Dioxide

(a)

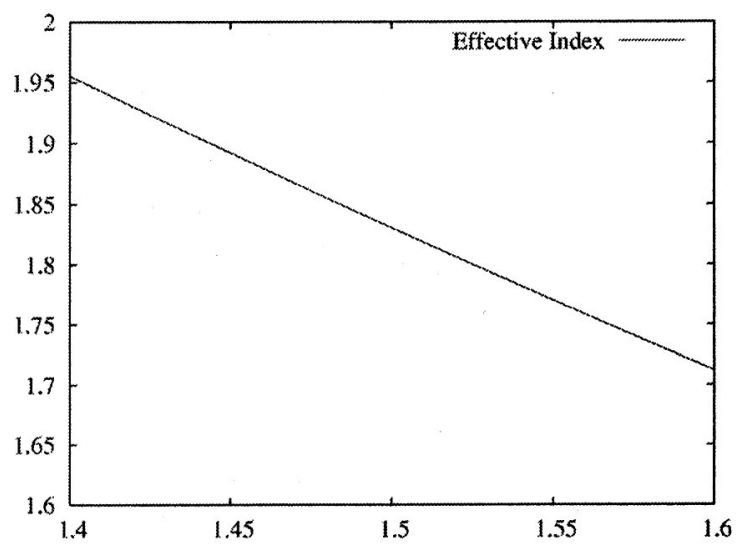

(b)

Fig. 1. (a) Diagram of the field pattern of the fundamental optical mode; contours of $|E|$ are plotted, starting at $10 \%$ of the maximum field value and incremented by $10 \%$ for each contour. (b) The dispersion diagram consists of the effective index plotted against the free-space wavelength.

\section{BLOCH-MODE ANALYSIS}

The analysis of the waveguide layout presented in Fig. 2 can be performed with the aid of Bloch's theorem. Our analysis begins with the conversion of the current-free and charge-free Maxwell's equations into an eigenvalue problem, following ${ }^{7}$

$$
\nabla \times \frac{1}{n(r)^{2}} \nabla \times H=\frac{w^{2}}{c^{2}} H .
$$

We choose the $H$ field as the field variable, because the eigenvalue equation is, in such a case, Hermitian and not generalized. We shall discuss the practical method of solving this equation for our structures, but first we will make some general observations about the solutions.

In what follows, we will take $x$ and $y$ to be the transverse cross sections of the waveguide, and $z$ is the direction of propagation. A nonsegmented waveguide, such as shown in Fig. 1, $n(r)$ has continuous symmetry in $z$. In the case of the segmented waveguide, $n(r)$ has the discrete periodicity $n(r+\Delta z)=n(r)$, where $\Delta z$ is a translation in the $z$ direction of the period of the segmentation. Utilizing Bloch's theorem in the propagation direction, we have the result that all the eigenvectors of Eq. (1) may be written in the form

$$
\Psi(w)=\phi(r) \exp (i \beta z) .
$$

Here we are taking $\Psi(w)$ as an eigenvector corresponding to a particular choice of $w, \beta$ as the crystal lattice vector, and $\phi(r)$ as the local-field distribution in a unit cell (a 3 -vector). The propagating modes of a segmented waveguide will be among the solutions to Eq. (2).

To solve this problem for complex structures, it is necessary to discretize it. There are many approaches available; we choose the finite-difference time-domain grid as the basis of the discretization in that such an approach implicitly enforces the appropriate continuity and divergence conditions. ${ }^{8}$ The resulting linear system results in a large sparse matrix equation, with approximately 0.6 million variables for a typical calculation, with a discretization of $0.02 \mu \mathrm{m}$. The lowest nonzero eigenvalues can nevertheless quickly be found with the proper choice of iterative methods. ${ }^{9}$ The modes generated by our direct solver were found to be identical to the modes generated by a large finite-difference time-domain simulation with a long runway and were substantially faster to generate.

It is important to ensure that Eq. (1) remains Hermitian in whatever set of boundary conditions are chosen for the unit cell. Although the $z$ boundary condition for a unit-cell calculation is obviously just periodic with the appropriate Bloch factor, it is less clear how the $x$ and $y$ boundary conditions should be handled. We wish to deal with the infinite-space problem, but for numerical calculations this is impossible. Instead, it is best to enforce zero-field boundary conditions on the edge of the unit cell in $x$ and $y$, corresponding roughly to having a perfect conductor in this region. Such boundary conditions obviously do not correspond to the problem at hand, but, for solutions to Eq. (1) that reduce close to zero at these boundaries, the introduction of this spurious conductor should not disturb the eigenvalue or vector.

One can define an effective index for the Bloch modes as $\beta / w$. With a choice of effective indices in the range of 1-4 for cells of periodicity $0.3 \mu \mathrm{m}$, the lowest-frequency eigenvalues turn out to be the fundamental propagating optical mode that has frequencies in the range of $1-2 \mu \mathrm{m}$. Thus solving for the modes of interest simply requires obtaining the lowest eigenvalue of the system for varying $\beta$ values. There is no reason this will be true all the time; in general, solving Eq. (1) with functions of the form (2) will produce a series of frequencies, only some of which will be in the range of physically meaningful solutions. It may be

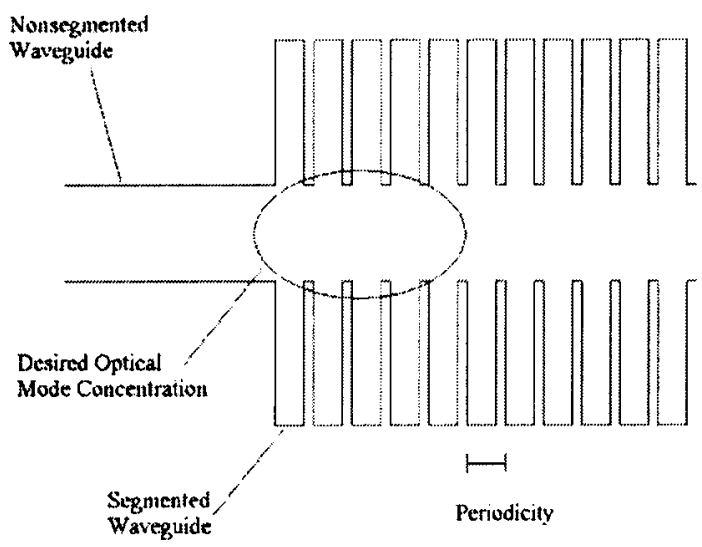

Fig. 2. Logical diagram of the planar layout of a segmented waveguide. For comparison, a nonsegmented waveguide is also diagrammed, in a configuration suitable for butt coupling into the segmented waveguide. 
necessary in other circumstances to climb several values up the eigenspectrum before useful data will be obtained. It is possible to generate a dispersion diagram for the Bloch modes of a given segmented waveguide design, by use of the effective index defined as above. The fundamental Bloch mode of a segmented waveguide with periodicity of $0.28 \mu \mathrm{m}$ and a duty cycle of 0.5 was solved for. We will refer to this design as series 0 , for later comparison with other designs. The dispersion diagram is presented in Fig. 3 , along with a transverse and longitudinal cross-section plot of the $E$ field intensity.

The dispersion plot in Fig. 3 indicates that the effective index of the Bloch mode closely matches that of a nonsegmented waveguide, indicating that there will not be much insertion loss from index mismatch if we couple light from a nonsegmented waveguide to a segmented waveguide. Moreover, there is little dispersion in the region of 1550 $\mathrm{nm}$ for this waveguide. This is not always the case, as many segmented waveguide periodicities can exhibit bandgaps with high backreflection and prevent the forward propagation of particular frequencies altogether. In the region of such behavior, the dispersion diagram typically exhibits a derivative $\mathrm{d} w / \mathrm{d} \beta$ approaching zero. Finally, it should be noted that, for our chosen periodicity, the field amplitudes become small at the edges of the waveguide domain. Again, unfortunate choices of periodicity may not exhibit this behavior and will exhibit higher radiative losses, and it is necessary to correctly design the segmented region to ensure low losses. Because the mode exhibits low loss in the modeled geometry, the segmentation need not be extended to infinity, and it is adequate to ensure that it is longer than several $e$-folding lengths of the field to prevent the optical mode from being influenced by the end of the lateral segments. For the devices fabricated here, the segments were extended $2000 \mathrm{~nm}$ from the center of the waveguide.

\section{FABRICATION AND TEST}

The devices were fabricated with electron-beam lithography. Wafers of SOI with approximately $120 \mathrm{~nm}$ of silicon for the top layer and $1.4 \mu \mathrm{m}$ of oxide were cleaved and were spun and cleaned in acetone and isopropanol. Dow Corning's FOX-12 electron-beam resist was spun onto the surface at $8000 \mathrm{rpm}$ and baked for $20 \mathrm{~min}$ at $180^{\circ} \mathrm{C}$. The wafers were exposed at $2250 \mu \mathrm{C} / \mathrm{cm}^{2}$ at $100 \mathrm{KV}$ in a Leica EBPG-5000+ electron-beam lithography system and developed in Clariant AZ300 metal-ion-free tetra-methyl ammonium hydroxide developer. They were rinsed in water, dried, and then etched in an Oxford Plasmalab 100 inductively coupled plasma, reactive ion etch system for 25 s with chlorine flow of 80 SCCM (SCCM denotes cubic centimeters per minute at STP), forward power of $50 \mathrm{~W}$, chamber pressure of 12 mTorr, and ICP power of $800 \mathrm{~W}$. Figure 4 shows a scanning electron micrograph of an exemplary device.

Couplers were constructed to couple light into nonsegmented waveguides directly from fiber arrays, ${ }^{1}$ and a tunable laser with a range from 1450 to $1600 \mathrm{~nm}$ and power of $10 \mathrm{~mW}$ was used to measure transmission spectra of these devices. Many different types of segmented waveguide were studied, which will be described further in

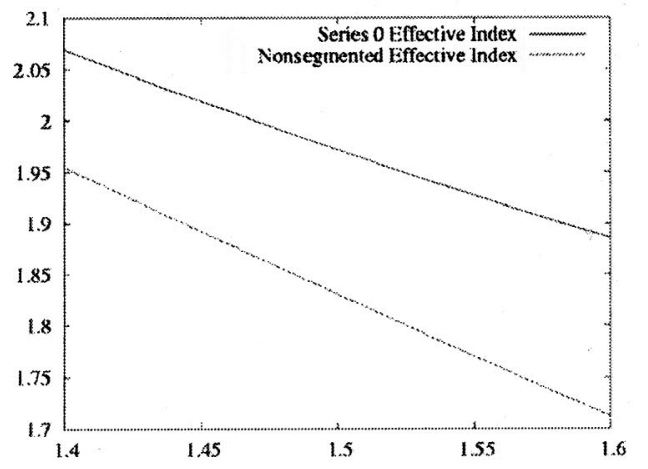

(a)

$1 \mathrm{um}$

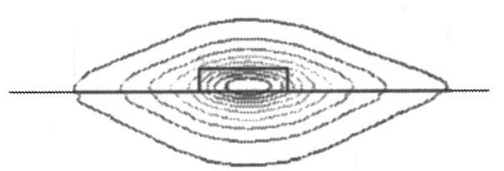

(b)

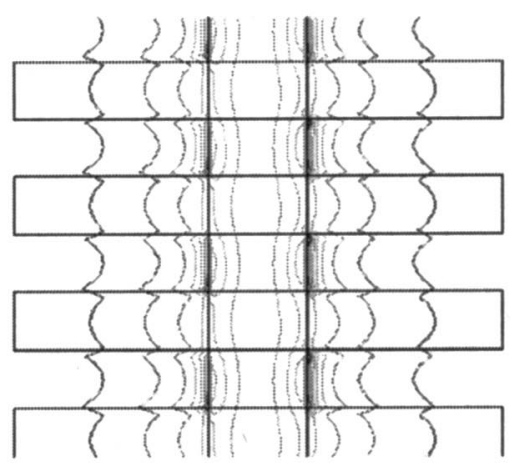

(c)

Fig. 3. (a) Dispersion diagram of both the series 0 segmented waveguide and the normal, unsegmented waveguide. (b) Modal patterns of the Bloch mode, with contours of $|E|$ plotted, starting at $10 \%$ of the maximum value and with contour increments of $10 \%$. The first plot (a) is on a $z$ plane that intersects the middle of a segment. (c) A plot on a horizontal plane that intersects the silicon layer halfway through. For clarity, four periods of the waveguide are shown.

Section 4. For each type of waveguide, we measured the waveguide loss of the segmented waveguide as well as the insertion loss induced by coupling from nonsegmented to segmented portions of the waveguide. For each design, 300 devices were fabricated with varying numbers of segmented-to-nonsegmented coupling interfaces and varying overall lengths of segmented waveguides. Measurement of these resulted in linear fits on the data that can be used to extract the frequency dependence of coupling and waveguide losses.

\section{RESULTS}

Data were taken for six different segmentation designs. Three parameters were varied: periodicity, duty cycle, and center size (i.e., the size of the unsegmented region of 
Table 1. Listing of Segmented Waveguide Series Studied and Essential Parameters at $1.48 \mu \mathrm{m}^{a}$

\begin{tabular}{lllccc}
\hline Series & $\begin{array}{c}\text { Periodicity } \\
(\mu \mathrm{m})\end{array}$ & $\begin{array}{c}\text { Duty } \\
\text { Cycle }\end{array}$ & $\begin{array}{c}\text { Center } \\
(\mu \mathrm{m})\end{array}$ & $\begin{array}{c}\text { Insertion Loss } \\
(\mathrm{dB})\end{array}$ & $\begin{array}{c}\text { Waveguide Loss } \\
(\mathrm{dB} / \mathrm{cm})\end{array}$ \\
\hline 0 & 0.28 & 0.5 & 0.5 & $-0.303+/-0.007$ & $-25.5+/-0.7$ \\
1 & 0.28 & 0.5 & 0.4 & $-0.569+/-0.004$ & $-40.4+/-0.6$ \\
2 & 0.28 & 0.65 & 0.5 & $-1.04+/-0.03$ & $-39.2+/-1.1$ \\
3 & 0.28 & 0.8 & 0.5 & $\mathrm{X}$ & $-16.3+/-0.4$ \\
4 & 0.28 & 0.35 & 0.5 & $\mathrm{X}$ & $\mathrm{X}$ \\
5 & 0.34 & 0.5 & 0.5 & $\mathrm{X}$ & $\mathrm{X}$ \\
\hline
\end{tabular}

${ }^{a}$ Devices that did not appear to support a mode (no light transmission observed) have X's marked for their measurements.

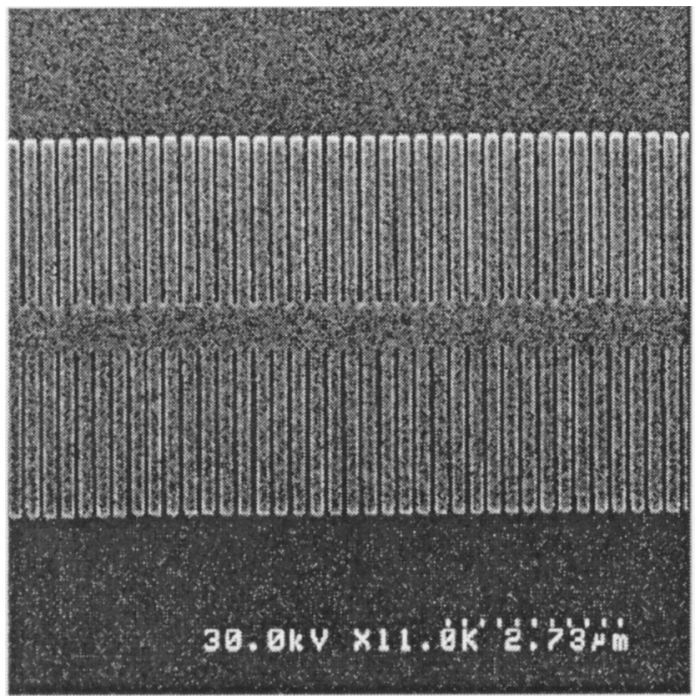

Fig. 4. Scanning electron micrograph of the series 0 segmented waveguide.

waveguide in the center). Table 1 lists the different parameters studied, as well as the measured waveguide loss and insertion losses for nonsegmented-to-segmented butt coupling at $1485 \mathrm{~nm}$.

A well-contained Bloch mode was found in this series of tests, and this low-loss optical mode can propagate with relatively low insertion losses from butt coupling from nonsegmented to segmented waveguide regions. Furthermore, it should be noted that other designs do not appear to support light propagation at all. These cases can be predicted from the Bloch-mode solutions. In the case of series 3 , for instance, a solution to the Bloch mode reveals a dispersion diagram similar to that of series 0 , but that mode would not be well contained. The $E$ field of such a mode would stay above $60 \%$ of its maximum value at all boundary conditions, regardless of the distance from the waveguide core to the boundary. The insertion loss of coupling from nonsegmented to segmented waveguides was also studied for series 0 with the finite-difference time domain. The results are plotted along with the frequencydependent insertion losses in Fig. 5.

The discrepancy between the simulated and observed results is actually quite small, differing by approximately $0.15 \mathrm{~dB}$. This translates into approximately a $1 \%$ difference in field amplitudes, which indicates excellent agreement between theory and experiment.
The full frequency dependence of the waveguide loss is shown in Fig. 6. It is important to note that it is relatively flat in frequency. The loss that is observed is comparable with the waveguide loss that has been measured ${ }^{1}$ in such a structure for a nonsegmented waveguide, or approximately $-7 \mathrm{~dB} / \mathrm{cm}$. The additional loss can be attributed to fabrication errors within the more complex periodic segmented waveguide, as well as to more substrate leakage predicted for the specified geometry. As substrate leakage is heavily dependent on the particular mode pattern, it is not surprising that there would be more such loss from a segmented waveguide compared with a normal waveguide. Moreover, this is the most probable explanation for the differences in losses between different segmented waveguide designs.

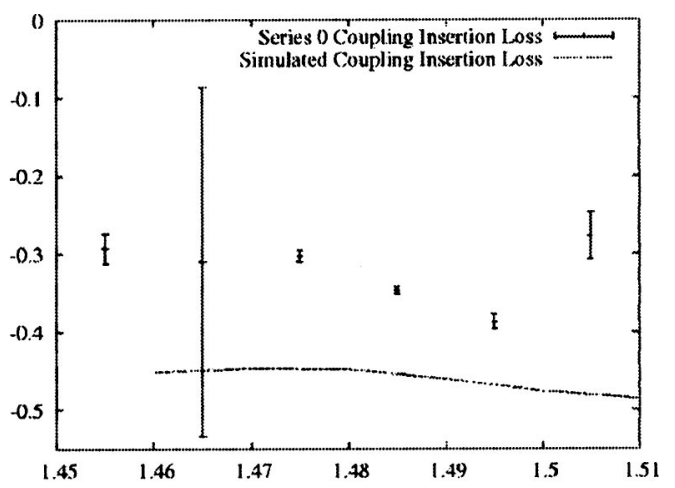

(a)

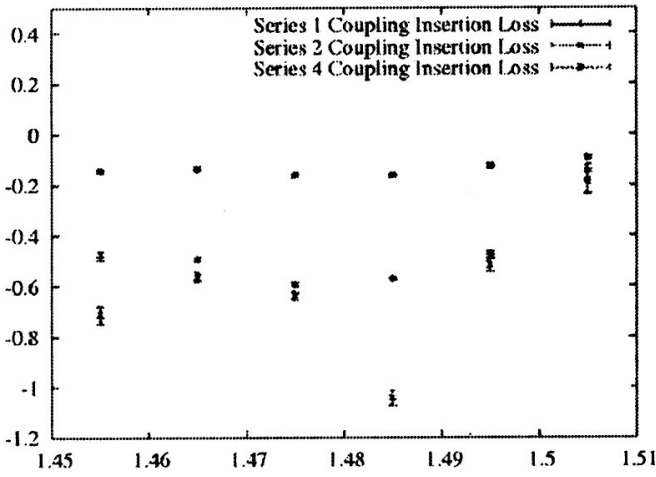

(b)

Fig. 5. (a) and (b) Measured coupling insertion loss for the segmented waveguide designs studied, in decibels, plotted against the free-space wavelength in micrometers. Series 0 , in (a), shows simulated data, which are presented for comparison. 


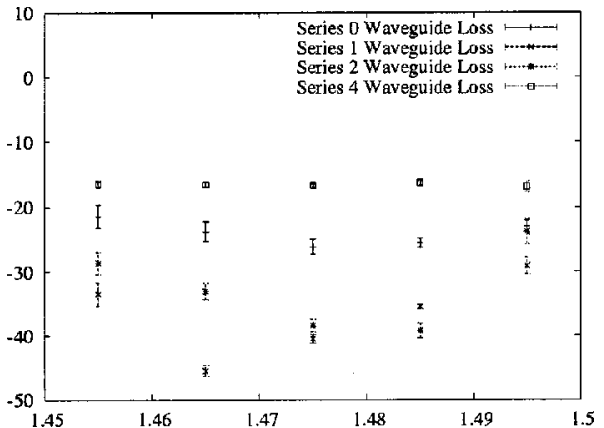

Fig. 6. Measured waveguide loss in decibels per centimeter as a function of the free-space wavelength for various segmented waveguide designs.

\section{CONCLUSION}

We have demonstrated that some segmented waveguide geometries can support relatively low-loss optical modes within thin SOI films. Moreover, these modes are quite easy to couple into from nonsegmented waveguides, exhibiting surprisingly low loss for simple butt coupling. We believe that this will permit segmented waveguides to offer viable options for electrically contacting optical waveguides in a single-layer structure. Also note that segmented waveguides offer broader applications beyond simply allowing electrical contact. For instance, one could employ segmented waveguides as low-loss frequency filters or as mode expanders by varying the periodicity of the grates. $^{10}$ Because the optical modes in these waveguides were supported in a broadband pattern, such a configuration might be useful in isolating a signal band from a pump wavelength in an optical system.

\section{ACKNOWLEDGMENTS}

The authors gratefully acknowledge the support of the Defense Advanced Research Projects Agency under the Chip Scale-Wavelength Division Multiplexing program, contract N00421-02-D-3223. Luxtera, Inc., is gratefully acknowledged for its donation of computer time.

T. Baehr-Jones, the corresponding author, can be reached by e-mail at thorolf@caltech.edu.

\section{REFERENCES}

1. T. Baehr-Jones, M. Hochberg, C. Walker, and A. Scherer, "High $Q$ resonators in thin silicon-on-insulator," Appl. Phys. Lett. 85, 3346-3347 (2004).

2. B. Maune, R. Lawson, C. Gunn, A. Scherer, and L. Dalton, "Electrically tunable ring resonators incorporating nematic liquid crystals as cladding layers," Appl. Phys. Lett. 83, 4689-4691 (2003)

3. Z. Weissman and A. Hardy, "Modes of periodically segmented waveguides," J. Lightwave Technol. 11, 1831-1838 (1993).

4. D. Nir, Z. Weissman, S. Ruschin, and A. Hardy, "Periodically segmented wave-guides in $\mathrm{Ti}: \mathrm{LiNbO}_{3}$," Opt. Lett. 19, 1732-1734 (1994).

5. Z. Weissman, "Evanescent field sensors with periodically segmented waveguides," Appl. Opt. 36, 1218-1222 (1997).

6. T. Baehr-Jones, M. Hochberg, and A. Scherer, "An analytic method for calculating substrate leakage in dielectric waveguides," J. Lightwave Technol., submitted for publication.

7. J. D. Joannopoulos, R. D. Meade, and J. N. Winn, Photonic Crystals (Princeton U. Press, 1995).

8. A. Taflove and S. C. Hagness, Computational Electrodynamis (Artech, 2000).

9. G. H. Golub and C. F. Van Loan, Matrix Computations (Johns Hopkins U. Press, 1996).

10. Z. Weissman and A. Hardy, "2-D Mode tapering via tapered channel waveguide segmentation," Electron. Lett. 28, 1514-1516 (1992). 